\title{
Reciprocal design: inclusive design approaches for people with late
}

\section{stage dementia.}

Gail Kenning,

Faculty of Arts and Social Sciences, University of Technology Sydney, Sydney, Australia

\section{Gail.Kenning@uts.edu.au}

Key words: Design for dementia, reciprocal design, inclusive design, fun, bridging

\begin{abstract}
More people, than ever before, are living into advanced old age. As a result incidences of agerelated, non-communicable disease such as dementia will increase. Dementia is an umbrella term for a range of conditions that impact cognitive and physical functioning. It is terminal.
\end{abstract}

Scientific and medical communities continue to search for a cure. Meanwhile, attention is increasingly being refocused and, rather than prioritizing a long life at any cost, exploring what it means to have a good quality of life and how to live well with dementia, regardless of the stage of the disease.

Design has an important role to play in supporting quality of life of people living with dementia. However, designers need to take into account the many varied contexts in which people live, the different manifestations of the disease, and individual wants, needs and preferences. Co-creative approaches can enable people living with dementia and stakeholders in their care, to engage in the design process and impact products and services made for them, provide opportunities for 
social engagement, interaction and pleasure, and give designers insights into the embodied experience of living with dementia and the social and cultural impact.

This paper discusses a participatory design research project with people living with advanced dementia in residential care facilities in Australia.

\section{Introduction}

The rise in the median age of the population, the number of people living into advanced old age, and the predicted increase in incidences of dementia, all have economic and health care implications, globally. Clinical and medical care and health care, respectively, are moving towards patient-centred and person-centred approaches. Advocates in dementia care are increasingly focusing on the importance of recognizing the person first, not the disease. With an increased focus on maintaining the personhood and selfhood of people living with dementia, there is a growing recognition of the need for the personalization of health care, and of everyday products and services that support people living with dementia, and stakeholders (family members, carers, care staff) in their care.

Developments in design and design research run parallel to the changes taking place in clinical medical and health care. Designers are increasingly recognizing the need for personalization in the day-to-day, employ user-centred approaches to gain input from users to ensure that designs meets their needs, and use inclusive methodologies that ensure vulnerable groups, such as those living with dementia, have their say. But, personalization can be time intensive and with growing needs in relation to ageing and dementia more designers are needed to work in this area. Alternatives are also needed to the one-on-one approaches, often used in the development of 
highly personalized outcomes (Hendriks et al. 2014; Kenning 2017; Kenning and Treadaway 2018; Maldonado Branco, Quental, and Ribeiro 2017; Treadaway and Kenning 2015).

In designing for dementia, designers need to gain a holistic understanding. This means gaining insights into the embodied experience of living with the disease, and so much can be learned from engaging directly with people living with dementia and stakeholders in their care. But, participatory design research projects need to be carefully managed to ensure reciprocity; that participants directly gain from the experience. Furthermore, because of the number of participants and designers often involved, logistics of time and location, and limited budgets, mean that it is not always possible for all participants to be in the same geographical location. How then can co-design practices negotiate these constraints?

This paper reports on an inclusive design approach for people living with advanced dementia and stakeholders in their care. The study employs mixed methodologies and draws on ethnographic approaches, arts engagement practices, communication theories, co-design research theories and practices, and design approaches for people living with dementia (Dervin and Foreman-Wernet 2013; Hendriks et al. 2014; Kenning 2016; Maldonado Branco, Quental, and Ribeiro 2017; Stephens, Cheston, and Gleeson 2012; Treadaway and Kenning 2015). The pilot, design research project enabled people living with dementia to have input into the design process, regardless of their abilities and level of contribution. It focused on the potential for reciprocity in participatory design approaches to ensure that it was mutually advantageous for all involved. The design process did not require participants to 'future think' design outcomes, which can be difficult for some people living with advanced dementia, but offered participants the opportunity to engage 'in the moment' and to experience 'in the moment' pleasure. The process also ensured that 
designers benefitted from the input of participants by gaining insights through observation and post-event analysis of the embodied experience of living with dementia. The project explored how inclusive design practices can enable a greater number of designers to engage with more people living with dementia, and how access can be broadened to reach more people (geographically) who can benefit from the design process and social engagement it offers.

\section{Background}

To understand the context in which this project was undertaken it is useful to review how health care is undergoing significant change, in no small part, due to the ageing population; the climate of personalization occurring in both health care and design; and the shifts in the hierarchies that have existed in some areas of design that means design processes can facilitate the inclusion of vulnerable groups, such as those living with advanced dementia.

\section{Context - Ageing population, dementia, and person-centred approaches}

Social and economic advances in living and working conditions, improvements in clinical and medical, and health care, and scientific and technological successes, have resulted in more people than ever before living into advanced old age (Prince et al. 2015). The median age of the global population is rising and the percentage of the population in their later years is larger than at any time before (Prince et al. 2015). Many people will grow old in relatively good health (Renehan et al. 2012). But, as people live longer there is a greater likelihood that they will experience periods of ill-health and, because there are more older people than ever before, the incidences of age-related non-communicable diseases, such as dementia, will rise (Prince et al. 2015; World Health Organisation 2017). Currently there is no cure for dementia (Prince et al. 2015). 
The ageing population and increased incidences of dementia has economic implications ('Australia to 2050' 2010; AIHW 2013; United Nations 2013; World Health Organisation 2017). Health and welfare resources are limited and so past and current levels of clinical and medical care for older people is not sustainable in most societies (World Health Organisation 2017, 17). In response to the demands of the ageing population and the limited resources available, changes are taking place in health care. These changes are also driven by a fundamental philosophical shift towards consumer driven care, a focus on supporting self-managed care, the maintenance of general wellbeing, and increased interest in non-clinical and non-medical approaches.

Person-centred approaches were developed in psychotherapy, and taken up first in dementia care, and then translated into aged care, and health care in general (Manthorpe and Samsi 2016). As a result some clinical and medical solutions offered in the past are no longer considered appropriate (World Health Organisation 2017). For example, expensive pharmacological solutions that have been offered in response to perceived behavioural problems are being replaced with holistic, inclusive, and person-centred approaches that offer socio-cultural alternatives (Mitchell, Dupuis, and Kontos 2013). To this aim, health care is not only addressing the desire for a long life (as has been the focus of much science and medicine) but, also focusing on how to achieve the best quality of life possible, including how to live well with chronic disease (Alzheimer's Society 2016a; National Health Scotland 2016).

The care of people living with dementia has undergone significant change and has led the way in terms of person-centred philosophies. Kitwood (1997) called for dementia care to recognize the person living with dementia as an individual with personal wants and needs, and for health care 
practices to respect dignity and personhood. This approach means acknowledging that people living with dementia cannot be addressed in terms of a shared pathology or as an homogenous group, but as a variety of people who undergo different experiences and have individual wants, needs, and desires (Harrison et al. 2007).

People living with dementia experience cognitive and physical limitations, and may experience explicit memory loss; the type of memory associated with people, places, and events (Harrison et al. 2007). While they may not be able to recall names, dates, and places, and may have difficulty carrying out tasks that they once did unaided, they retain emotional memory through to end of life and can experience sadness, happiness, and enjoyment and fun (Sabat 2005, 2006). As people living with dementia undergo cognitive decline, they may not remember aspects of their own lives and be reliant on others to prompt them about people they have known, places they have been to, and occupations they have engaged in. Some forms of dementia impact on behaviour to such an extent that family members claim that they are 'like a different person' (Alzheimer's Society 2016b). Understanding the wants, needs and desires of people living with dementia means understanding who the person is, where they live, their day-to-day context, and their primary relationships. Understanding who they are may also involve finding out about who they have been in the past, their employment history, and their likes and dislikes from family members, carers, and care staff. However, it is important that information provided in this way does not become proxy for direct engagement with the person living with dementia who can, with consideration and care, engage and communicate even when verbal communication is no longer possible (Kontos and Martin 2013; Mitchell, Dupuis, and Kontos 2013; Sabat 2005, 2006). People living with dementia can engage in meaning making, and can have a good quality 
of life and opportunities for social engagement, and involvement in meaningful activities can contribute to positive wellbeing (Cutler 2009; Sabat 2005; Kenning 2016).

These changes in the context of care have implications for how design intersects with health care and wellbeing. Therefore, it is useful to examine the parallels that have occurred in relation to the development of design research and processes.

\section{Design, co-design, participation, design research, design for social change}

Recognizing the importance of acknowledging individual wants and needs foregrounds the importance of personalization in products, services, and care that support people living with dementia in the everyday. Recognizing that people's needs are ever-changing highlights the necessity for flexibility and adaptability (Kitwood 1997; Manthorpe and Samsi 2016). Furthermore recognizing, as Sabat (2005) suggests, that people with advanced dementia can engage in decision making, means that systems and processes need to be adapted and developed to ensure that everyone can engage and contribute to the best of their abilities.

Design and design research has much to offer people living with dementia and stakeholders in their care. As with clinical, medical and health care, design and design research have undergone cultural and philosophical transformations, and sets of theories and practices have evolved to embrace a multitude of viewpoints and approaches that are experiential, inclusive and participatory (Jonas 2014; Norman 2016; Rodgers and Yee 2015). For example, Norman (2016) traces the development of design as it transitioned from a focus on the object, to a force in industry, and subsequently offered new experiences and ways for people to interact. He engages 
in 'big picture' thinking by suggesting that design offers an 'evolution in[to] ways of thinking, [and] problem discovery', moving beyond the previously noted emphasis on materials and technologies to focus on experience and opens up ways 'to enhance the lives of individuals, the experience of the workforce, and even the health of the planet' (Norman 2016).

As discussed, there has been a refocusing of attention towards the end-user in many varied disciplines. In clinical and medical fields it involves a shift towards patient-centred and evidence-based care (McClimans 2017,vii-xv), in health care it involved embracing the concept of person-centred care (Chenoweth et al. 2014), and similarly, in design experiential and usercentred approaches have now come to the fore. However, these changes are challenging as, for example, the concept of co-creation raises questions about what has been the accepted role of the designer as the Auteur. As the design process is opened up - to varying degrees - to enable the user to have an input, the level of 'control' over outcomes becomes a process of negotiation. The level of participant input needs to be given careful consideration to ensure that it is appropriate to the overall aims and outcomes of the project. After all, the type and extent of the engagement required may differ considerably depending on whether the desired outcome is the production of an object, a service, a tool, an experience in itself, social change, or research (Bang and Vossoughi 2016; Jonas 2014; Brandt 2004).

As design has links historically with arts, crafts, science and technology, projects that engage with users need to negotiate the paradigmatic differences that currently operate in relation to design and design research (Jonas 2014). For example, Jonas (2014) traces the development of design research through the cultural and paradigmatic divides of the arts and sciences, and 
acknowledges the tensions that arise in relation to the ongoing hierarchies of theory and practice. He notes the (often judgmental) differentiations made between 'reflection', as associated with practice, and 'Research', which is all too often located with the concept of building knowledge through theory. But, Jonas brings us to a point of middle ground through transdisciplinarity, which embraces a multitude of approaches, and, importantly in terms of design research projects such as the one discussed in this paper, recognizes the importance of embodied and situated research that actively engages through participation (Jonas 2014).

As design approaches increasingly focus on 'experience' the role of the user becomes paramount. Co-creative, participatory approaches, therefore, enable potential users to take part in the development of products and services created for them. This not only challenges the role of the designer and the user, it also raises critical questions around the process of engagement, and who has access to it. This becomes particularly important in relation to marginalized and vulnerable group, such as those living with dementia. We see this issue addressed in participatory approaches and in particular Participatory Design Research (PDR), which developed in the field of pedagogy and education as a method and approach that focusses on the desire to effect social change and to re-address power dynamics (Bang \& Vossoughi 2016).

\section{Participation in design for dementia}

In relation to people living with dementia, participatory approaches that focus on personalization, and include them in the design process, facilitate engagement and address dementia, not as lack and deficit, but through a lens of possibility and potential. In doing so they challenge stigma, encourage access for vulnerable groups and can contribute to social change (Hendriks et al. 
2014; Maldonado Branco, Quental, and Ribeiro 2017). These participatory approaches attend to the ways in which normatively powered dynamics are re-inscribed in the roles and relations between researchers and 'the researched', and disrupt or create new roles and relations and to achieve, in Bang and Vossoughi $(2016,173)$ terms, transformative ends. In using such approaches, design for dementia can engage in design projects that advance fundamental knowledge of learning and development in relation to dementia, and become 'social change making projects' (Bang and Vossoughi 2016).

Whereas designers working in the area of design for dementia have often looked to pathological, clinical, and scientific understandings of the condition, increasingly salutogenic aspects are being explored (Mittelmark and Bauer 2017). Designers recognize that they can gain insights into the embodied experience of living with dementia through contextualized and situated research in the care environment. The very different embodied experiences of individuals, the relationships with carers, family, care staff, and clinical and medical staff are all brought into the picture, and different types of dementia, the variety of symptoms and conditions, and different living conditions are made apparent. Insights are gained from seeing how care is undertaken, using approaches that focus on embodied experiences, on the wants and needs of the individual, and how personhood is respected through person-centred and relational care (Kitwood 1997). In this way design becomes a research process to collect data for analysis that can inform a wider audience including dementia discourse, dementia studies, dementia care, and design for dementia (Kenning 2017; Kenning and Treadaway 2017; Treadaway and Kenning 2015). 
Co-design projects in this environment further challenge the role of designers and the relationship between the designer, design and the user (Maldonado Branco, Quental, and Ribeiro 2017). The level of input the user, or participant, can have is impacted by the overall design of the project. Consideration needs to be given to the extent to which it supports and facilitates user engagement and participation, particularly for users who may have specific access needs (Hendriks et al. 2014). The design of the project needs to ensure that it is not too long, confusing or tiring for people living with dementia, particularly those in the later stages of the disease, and that participants are not overwhelmed by the number of people they engage with during the design process. Co-design approaches also need to be able to accommodate participants who are not able to verbally articulate wants, needs, or desires; have cognitive and physical limitations; whose level of input, while important, may not be extensive; and those who may have difficulty with comprehending the role of probes and prototypes. Projects may be restrictive because, for example, they require the user to understand the design process and 'future think' the end product, or be able to explore concepts and possibilities, think about alternatives, or empathize with the designer (Bang and Vossoughi 2016).

\section{The impact of design for dementia on participatory approaches}

Personalized approaches are needed to support personhood, dignity and respect and recognize, that in relation to ageing and dementia, there is no single approach or 'one size fits all'. Projects that are well-designed, open, participatory, adaptive, and flexible can enable vulnerable users to engage in the co-design process; allow for personalization and reciprocity; and with adaptation, and change can be developed to suit the needs of people living with dementia, including those in the later stages of the disease (Hendriks et al. 2014; Maldonado Branco, Quental, and Ribeiro 2017; Treadaway and Kenning 2015). Such approaches in design, that focus on personalization 
and recognize the user as individual, can as Kitwood (1997) suggests in relation to dementia care, promote dignity and personhood, while also creating personalized products, processes and services that contribute to everyday experiences and improve quality of life. Well-designed cocreative, participatory approaches not only enable participants to be involved in the design process and contribute to the outcomes, they can also enable participants to benefit from the design process and the social engagement, interaction, sense of connection, fun and pleasure it brings (Kenning 2017; Maldonado Branco, Quental, and Ribeiro 2017).

\section{The study}

The design research project used an interpretivist approach following the rationale of good qualitative research in presenting 'a logical chain of reasoning, [and] multiple sources of converging evidence to support an explanation' (Suter 2012). The study used qualitative methodologies drawing on social and reflexive approaches such as action research and grounded practical theory (GPT) to understand how to co-design objects and activities for people living with dementia while also providing opportunities for social interaction and engagement during the design process (Craig and Tracy 1995; Denscombe 2008).

There were three levels of engagement in the design research project. Workshop participants included people living with dementia and stakeholders in their care, made up of family members, carers, care staff, and management from The Whiddon Group — the care provider organization of the residential aged care facilities (RACF) in the project. Design researchers experienced in design and research methodologies (including the project lead), engaged directly with participants in the workshops, and the designers in the 'meet ups'. They collected and analysed data and were involved in the production of prototypes alongside the designers. Designers, with 
experience in product design, textiles, industrial design, art, and technology, worked at a distance from the workshop participants, but engaged directly with the design researchers as they all developed prototypes that were explored and developed in subsequent workshops.

Workshop participants were recruited using a qualitative purposive methodology that focused on 'information-rich cases yielding insights and in depth understanding rather than empirical generalizations' (Patton 2015, 230). Two RACFs were selected for inclusion in the study following discussion between researchers and care staff, Directors of Nursing, and the executives at the care provider organization. One RACF was selected in South West Sydney and another, for its potential to offer different perspectives, was in a small regional town in Northern NSW a 6-hour drive (or 70 minute flight) from Sydney. People living with dementia were selected following discussion between RACF care staff, family members or legal guardians, and researchers. They included six females and two males (four residents from each RACF) between the ages of 66 and 96 . The criteria for selection was that they had a diagnosis of advanced dementia; were able to engage in a workshop for a period of 1-2 hours; would benefit from engaging with people, objects and activities; were unlikely to experience undue stress or anxiety from participation; and that they were able to communicate verbally or non-verbally.

The RACFs gave researchers basic client data which included age; general health and wellbeing; type of dementia and comorbidities; names and relationship of family members; places lived; occupations; past and present interests and hobbies; and likes and dislikes. The amount of information varied from person to person and between RACFs. Consent for all participants was arranged in consultation with the RACFs who had access to family members and legal guardians, 
and participants living with dementia also gave their personal consent. Consent was reassessed on individual bases throughout the workshops using a process consent approach (Dewing 2007), which acknowledges that verbal and non-verbal behaviours and responses can be indicative of stress, anxiety, and concern and can constitute a withdrawal of consent. It also recognizes that consent may be reinstated if the participants exhibited behaviours and responses consistent with wanting to become involved in activities again.

Stakeholders were invited to participate if they were a family member or carer of a participating person living with dementia or a staff member or volunteer at either RACF who knew them. Designers were invited to take part following an open call through online and offline design networks. The criteria for designer participation was that they had formal training or experience of art or design practices or research; an interest in social design, designing for positive impact, or working with vulnerable groups (no prior experience of working with people living with dementia was needed); could commit time; had a willingness to engage in a co-design approach and contribute ideas, skills, and make prototypes.

The first research question explored the extent to which participants that included people living with advanced dementia can participate in workshops using co-design, participatory approaches, and how co-design workshops can provide opportunities for social interaction and contribute to the positive wellbeing of people living with advanced dementia. A second research question explored the extent to which co-design practices could be carried out when not all participants were in the same geographical location; could use minimal direction and be flexible and 
adaptable to enable people living with advanced dementia; and provide designers with insights that would encourage ongoing work in this area.

Ethics approval was granted through the Human Research Ethics Committee of the University of Technology Sydney (HREC 2015000330). The study used unstructured interviews; workshops and 'meet ups'; q questionnaires; basic demographic and health data; participant surveys in each workshop; designer questionnaires, completed at the end of the project; photographs; audio and visual probes; and prototypes as they were developed throughout the project. The designer 'meet ups' took place after the first workshops at the RACF had been held, and continued on a weekly basis. The workshops and follow-on discussions were audio and video recorded to enable postevent analysis. Design researchers made direct observations throughout the workshops and kept journal notes. An observation schedule was used as a starting point for the design researchers. The observation schedule, a modification of schedules used in the evaluation of arts engagement programs, listed positive and negative affect states (happiness, sadness, interest etc.), and examples of indicators (possible bodily responses) that reflected these states (Kinney and Rentz 2005; Lawton, Van Haitsma, and Klapper 1996). Unlike many studies that use observation schedules, the design researchers in this study observed participants throughout the entire workshops (not for intermittent periods). Furthermore, they did not allocate scores to, or count duration of affective responses, but were trained in how to use the observation schedules to describe and record the intensity and frequency of responses in text; in line with deep qualitative research approaches (Cutler 2009, 344). Once they had a good understanding of the observation requirements, design researchers chose to make freehand notes rather than record information on 
the observation schedule. A design researcher debrief meeting was held, and recorded, at the end of each workshop and design researchers continued to make notes in the following days.

A key aspect of working in design for people living with dementia in residential facilities is the need for flexibility and the ability to adapt the study according to circumstances. The workshops were conducted in very different circumstances in each of the two RACFs. In the first, the workshops took place in a small television room, with soft furnishings. Only workshop participants were in the room, which allowed for attention to be focused on them. However, the room was not conducive to all participants consistently engaging with each other and small selfcontained 'niche' groups emerged. The workshops in the second facility took place in the breakfast room, which was also used for creative activities. The room had good lighting and large tables, which meant participants could interact as a single group. However, a number of people not involved in the workshop were also in the room, sat at other tables. While this did not distract participants, it meant that careful consideration needed to be given to the audio and video recordings to ensure that only those who had provided consent were visible and audible on the recordings. In addition, some onlookers expressed a desire to be involved in the workshop activities, and the design researchers needed to accommodate this. 


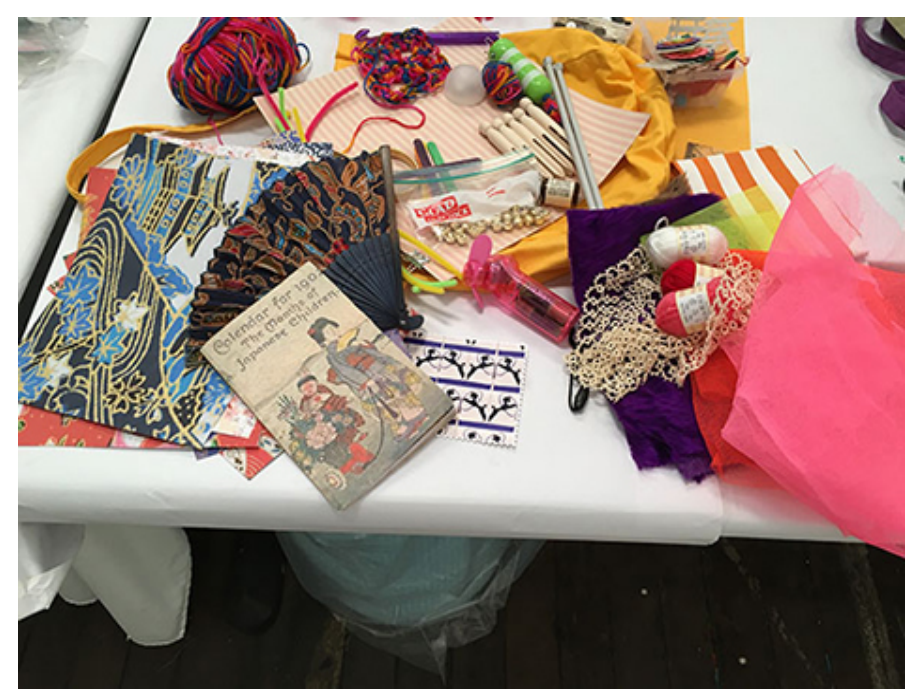

Figure 1. 'Akemi's' 'fiddle bag' contained objects, to reflect her cultural heritage

The first workshops conducted with people living with dementia and stakeholders in their care provided opportunities for participants to engage with and respond to a range of visual and tactile stimuli and cultural probes, and with each other. The workshops ran for two hours and began with a conversational introduction to the morning's activities, a brief overview of the design research project, and a period of form-filling as participants completed short questionnaires. Each person living with dementia was given a personalized 'fiddle bag', consisting of a strong canvas bag containing an array of materials, objects and activities that had been selected for each of the individual participants in the workshop (Figure 1). They included, for example, textile fragments; threads; nuts; bolts; children's clothing; knitting needles; images of weddings and cars; music; aromatic oils and an array of materials that could promote an affective response, such as, laughter and joy, or prompt a memory or story. The 'fiddle bags' were designed to be fun activities and provide participants with the element of surprise as they took objects out of the bag for further investigation - earlier research had found that the act of putting things in or taking them out of bags or pockets stimulated curiosity and pleasure (Kenning and Treadaway 2017; Killick and Kenning 2015). A family member or care staff sat with each participant as they 
looked through the 'fiddle bags', emptied out the contents, and commented on each of the objects, or engaged in storytelling (Figure 2). Design researchers interpreted responses through close observation, which was particularly important in relation to those who were not verbally articulate. Participants were able to explore the 'fiddle bags' at their own speed, discard items by moving them away or giving them to other participants, return to items previously rejected, and make claims to objects that other participants did not want. The accompanying family member or care staff made notes in booklets and on the paper tablecloths provided about what objects were liked and disliked. Design researchers observed participants' responses to the materials, objects and activities, and assessed the extent to which they were liked or disliked; encouraged interaction with other participants; or stimulated discussion, storytelling or an affective response such as laughter. They were also able to gain an understanding of physical and cognitive abilities through observation and interaction with participants. These observations and findings were analysed and given to a group of designers and used as the basis for the development of a series of prototypes made for the workshop participants to encourage, laughter, play and fun, or to occupy and entertain.

After the first workshops, nine artists, designers and technologists (designers) from in and around Sydney were invited to attend a 'meet up'. They were given an overview of the pathological understandings of dementia; the impact of dementia on individuals and stakeholders; the implications of dementia for society; and provided with academic and industry research. Details about workshop participants and the workshops was 'bridged' to designers who were provided with details about what participants said; how they responded; design researchers' observations; details about participants' abilities or limitations; and the level of fatigue 
experienced by participants in the workshops. In addition, designers were given access to all visual and tactile materials from the 'fiddle bags'. The first 'meet up' was followed by a series of weekly 'meet ups' over a four-month period in which a series of prototypes were developed based on the information provided.

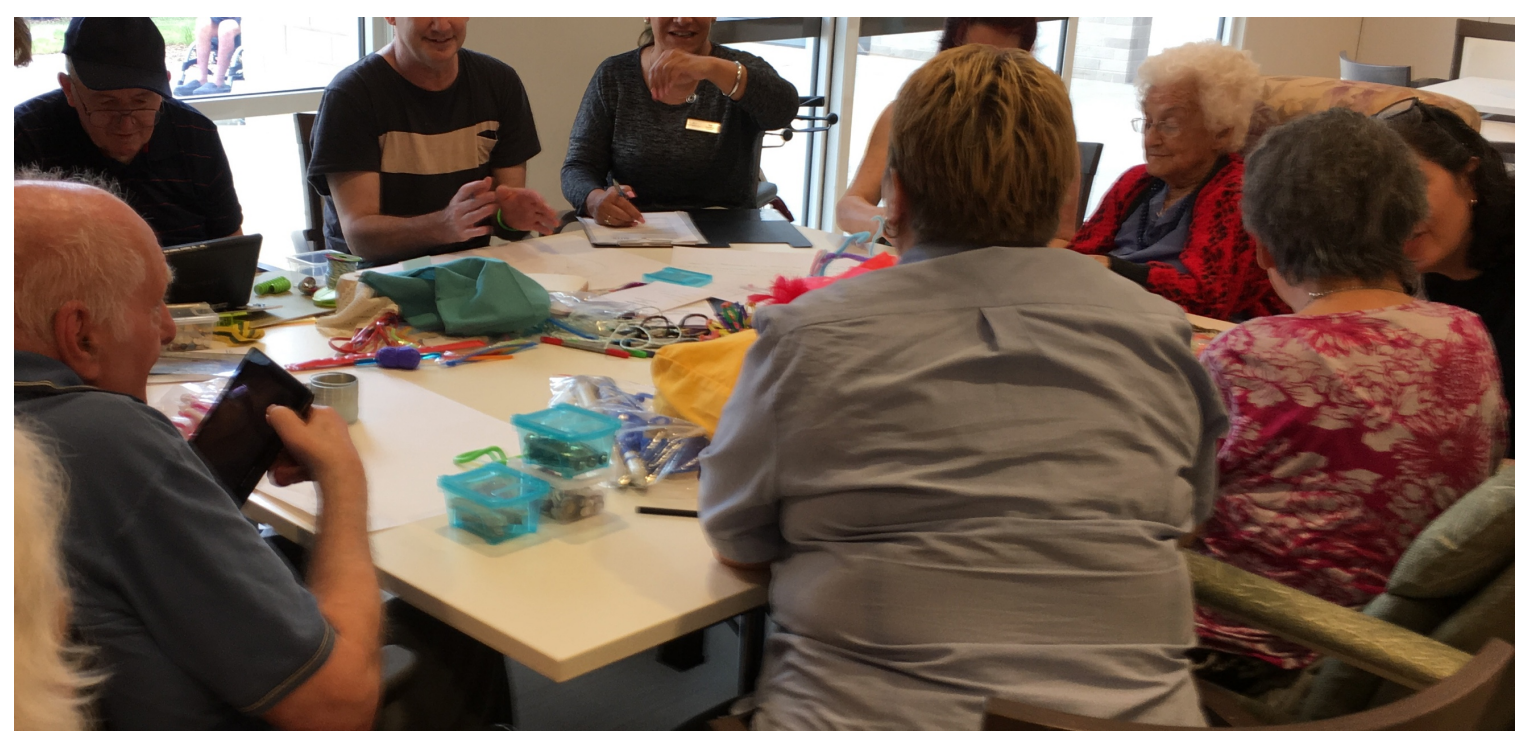

Figure 2. Participants shared objects from each other's 'fiddle bags, as they engaged with each other and family members and care staff'

In the second workshops participants were given the prototypes made by the designers during the 'meet ups'. As with the first workshop, minimal direction was given to participants. Family members and carers sat with participants with dementia as they engaged with the prototypes, with each person being given access to each prototype. Participants were invited to interact with the objects and activities; provide feedback; show whether they liked or disliked them; talk about them; and asked about what changes they would make. Family members and care staff made notes about the experience in booklets provided and researchers observed and analysed the process. The information from the workshop was 'bridged' to designers in the form of observations and analysis, and images and video, which were all made available to designers at the 'meet ups' to enable them to further develop the prototypes. 


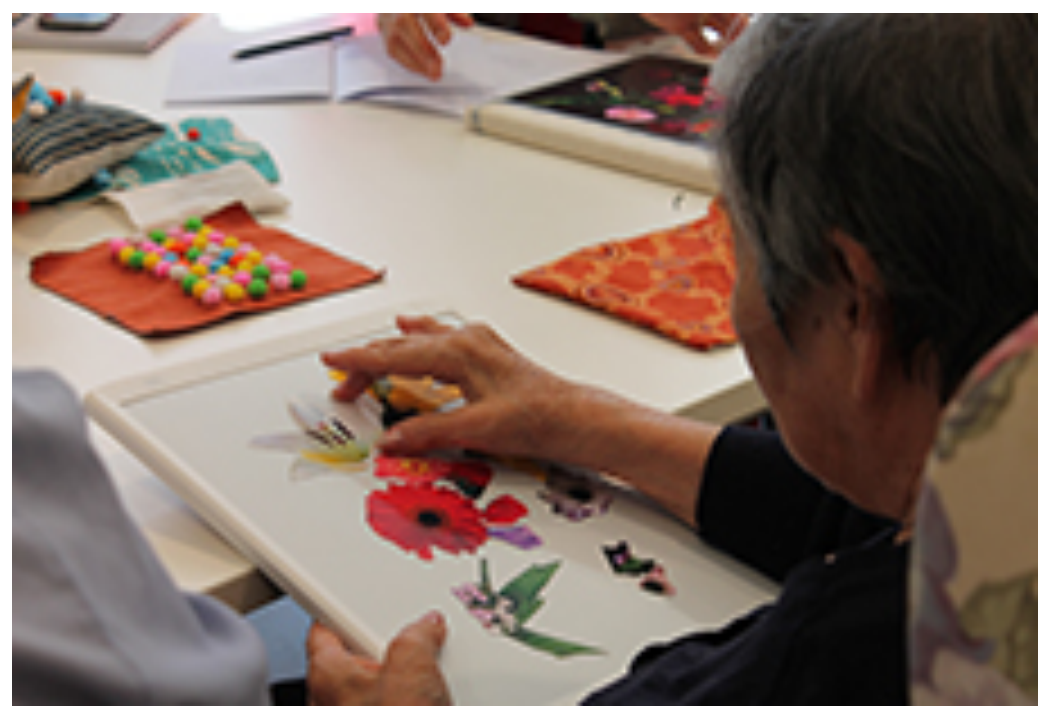

Figure 3 'Akemi' engaged studiously with each of the prototypes

The third and final workshops explored the extent to which participants engaged with the advanced prototypes and final products made by designers using data provided to them from workshops one and two, and the extent to which the prototypes brought pleasure or facilitated engagement with others. Participants were asked to engage with and critique the artefacts and to provide information and feedback on their level of interest in them.

The findings from all workshops underwent thematic analysis. Data was analysed using Word, Excel, Nvivo version 10, and video analysis software. Inductive themes were established prior to the stakeholder workshops, based on existing academic and industry design research literature relating to participants with dementia. These were given to researchers as part of the basis for their observations. Researchers also identified emerging themes in follow up discussion after each of the stakeholder workshops and during the designer 'meet ups'. In addition, deductive themes were drawn from the analysis of speech, gestures, the observation schedules and notes, design researchers' notes, and unstructured interviews and discussion before and after the 
workshops. Analysis of the themes took into account verbal and non-verbal responses and their context, including the physical abilities and type of dementia of the person and what or who prompted the response. This provided evidence of the impact of the engagement and insights into individuals 'in the moment' pleasure and wellbeing. The array of themes will not be discussed in this paper, but are available (Kenning 2017).

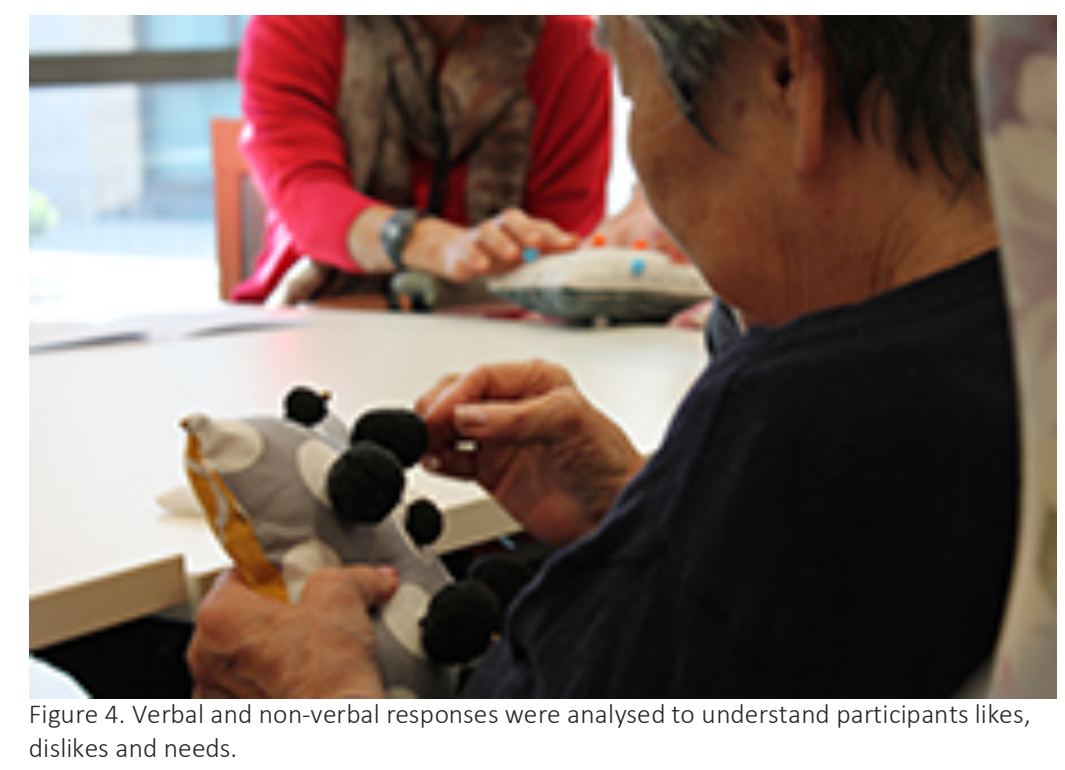

The prototypes that were developed were provided to all participants in the workshops and openly discussed during the workshops and in the 'meet ups'. Some prototypes were developed by designers working together and others were championed by individual designers who followed the work through from inception to completion, making changes based on the input from the workshop participants. The range of prototypes completed included a Fashion Studio Kit that enabled fashion outfits to be assembled from a range of miniature clothing items made from fabrics and threads; Fiddle Blankets with pom-poms, beads or buttons to be grasped and touched; Fiddle Cushions made from a range of fabrics with buttons, beads, and similar attachments; A Flower Arranging Kit with magnetic fabric flowers that could be arranged to make a picture; A Gear Kit made up of laser cut gears to be mounted on a solid wooden board 
using nuts and bolts, which once assembled the gears turned on an LED light; A Kumihimo Weaving Form which was a large hexagonal disk with printed instructions to remind users how to weave together six threads; A Music Box which was a small round padded box with five threads attached to a small microcontroller and a speaker; A Musical Cushion made from a range of fabrics embedded with pressure sensors connected via Bluetooth to software generating sophisticated music; A Peg Picture Kit using a series of dolly pegs and felt shapes enabling the user to assemble pre-designed pictures; A Quilt Picture Making Kit made from a series of fabric shapes with embedded magnets to be assembled on magnetic boards to create patterns; A Sorting Blanket consisting of a large central pocket containing small objects to be sorted and 10 pockets for the objects to be sorted into; and a Spoon Piano using Makey Makey TM to connect a set of dessertspoons to a laptop computer to enable users to play piano tunes.

\section{Discussion}

This paper will primarily discuss the structure and methodology of the project and the potential for this approach to provide access to the design process for people living with dementia. The project facilitated reciprocity through its open approach and minimal directing of participants, which allowed participants to interpret instructions and respond in whatever way was appropriate to them. It also employed a 'bridging approach' that enabled more participants and designers to be engaged in the project, by not requiring that all participants, design researchers and designers were involved in the same location at the same time, and allowed for involvement to take place at a distance.

The aim of the project was to use participatory approaches to engage people living with advanced dementia and stakeholders in their care in the co-production of a series of personalized 
objects and activities, which would encourage occupation, engagement or play, and for the design process to be an opportunity for engagement, connection, and pleasure. This approach sought to challenge the view that people living with advanced dementia could not actively engage in group workshop environments and co-production processes. The open and minimal directive approach taken throughout the workshops removed the need for 'future thinking' of the design objects, and enabled people living with advanced dementia, with limited verbal communication and cognitive and physical limitations, to engage with each other, enjoy the design process, and to contribute. The first workshop showed that people living with advanced dementia could engage with objects, materials and activities, as evidenced by them picking them up, exchanging them with others, talking about them, telling stories, and physically moving (without support of care staff or family) to gain access to them. In subsequent workshops participants engaged with prototypes, as they progressed, by taking them apart, assembling them, talking about them, and giving verbal feedback on what they thought should be changed. Importantly, it became apparent that some participants were able to 'future think' the design of the objects (to a limited extent) and could be asked about design improvements they would make, and how they would like the prototype to be changed or adapted. For those with verbal capacity, being asked their opinion on what should be changed gave participants a sense of importance and recognition and they did not hesitate in giving their views.

The approach used allowed all participants to engage with all objects and prototypes, whether part of their 'fiddle bag' or someone else's and whether they had been personalized for them or not. It was important that formalities were minimized and that minimal formal direction was given during the workshops, as this encouraged participants to engage at whatever level was 
appropriate for them. It also allowed for participants to change their mind in how they responded to objects, provided moments of normalcy (Kenning 2016). All responses were captured as data and analysed providing an indication of likes, dislikes, affective engagement, and emotional responses. The data analysis took into account, in the cross referencing of themes and contextualizing of responses, the range of physical and cognitive abilities, types of dementia, levels of energy and engagement over the period of the workshops, and the verbal and nonverbal communication abilities of participants.

The project recognized that not all participants were able to envisage the design project as a whole, or their part in the design process, or were able to 'future think' end outcomes. Therefore, the project focused on the concept of reciprocity in design research. This ensured that while designers both contributed to and benefitted from the design research process, the design process was pleasurable, and fun 'in the moment' for participants, and did not only focus on providing enjoyable outcomes at the end of the project—in the form of objects and activities to engage and entertain. Reciprocity was achieved as evidenced by the level of engagement observed and in the analysis of verbal and non-verbal responses.

The design research project worked with a range of experts distributed throughout the care system. However, it also needed to take into account how people living with dementia and stakeholders in their care can engage in participatory projects with experienced designers, when living in regional or remote locations. Bringing all designers and participants together in the same space was not feasible due to budget restraints. Therefore the 'bridging approach', of using two experienced design researchers in each workshop environment and seven designers in the 
'meet ups', was a workable and efficient compromise. These two groups engaged in ongoing exchanges about the participants in the workshops, about their lives, needs, and relationships. Videos and images from the workshops and the objects and activities in the 'fiddle bags' provided the designers with information needed to develop prototypes. The 'bridging approach' was adopted because of the geographical location of the RACFs. However, it became apparent that this approach could also be used to address the need for more designers in the area. Designers, even when they did not have direct access because of time, geographical location, or budget restraints, gained in-depth insights into people living with an embodied experience of dementia, and participants with dementia were able to take part in the workshop environment without being overwhelmed or made anxious by having too many people they did not know being in the immediate vicinity.

The focus on reciprocity and ensuring that participants enjoyed the workshops 'in the moment', the use of minimal direction with participants, and use of the 'bridging approach' drew upon Sense-Making frameworks, as used in communication theory and practices. This recognizes that responses made by participants are in an ongoing state of change and development, and are not fixed (Dervin and Foreman-Wernet 2013). It posits that people often need space and time to engage in open and honest communication, to share and compare responses, provide genuine feedback, and to resist influences that may inadvertently encourage compliance, consensus or agreement (Dervin \& Foreman-Wernet 2013, 153-55). Therefore, in this project participants and designers were given time and space to engage with and adapt their responses to material stimuli and other participants' responses over time. The approach recognized the importance of not only understanding the personal preferences and wants and needs of people living with advanced 
dementia, for whom objects and activities would be made, but also the context of how they live, their primary relationships, and their physical and social environment.

\section{Conclusion}

The importance of reciprocity in the design process has been well documented. When working with people living with advanced dementia this becomes an ethical consideration for designers working with vulnerable groups, who may not be able to understand the design process as a whole. The openness of the exchange between workshop participants, design researchers, and designers also shows the importance and ethical consideration of providing access by ensuring that the process enables participation according to ability, and does not provide limitations by adding complexity to the design process. The 'bridging approach' is not a replacement for direct access between designers and participants in co-design activities. It is preferable, when possible, for all to engage in the same space. But in this project, while not all designers engaged directly with people living with advanced dementia, the advantages were that participants in diverse geographical locations could be supported through design within a fixed budget; that designers could gain a holistic understanding of dementia and its impact on individuals and society, and insights into the embodied experience of individuals; and it ensured that participants engaged in an enjoyable, fun, social interaction without the fatigue or stress that can occur from engaging with large groups of unfamiliar people.

In thinking carefully through the design process, designers can use participatory design and research practices to address the power dynamics and hierarchies of legacy design practices that can inadvertently negate marginalized or vulnerable groups, such as those living with advanced dementia. Furthermore, design and design research has much to offer people living with 
dementia and stakeholders in their care in terms of products and services, and it can also contribute to dementia discourse by building awareness and challenging legacy perceptions of what it is to live with dementia and how limitations of the condition can be overcome. Importantly, the study showed that people living in advanced stages of dementia were able to participate in an inclusive group design process according to their individual abilities, which staff at the care facility reported as being unexpected and surprising. The 'Reciprocal Design' approach provided opportunities for social engagement, interaction with objects and activities, and entertainment for people living with dementia and stakeholders in their care. The study also showed that participants living with dementia, who were not able to verbally articulate likes and dislikes, could communicate in different way, which included unexpectedly moving around a table (without the aid of their walker) to engage with prototypes or objects with which they had become fascinated, or laughing and smiling as they engaged for sustained periods of time. Designers engaged in the project reported that they benefitted from the experience by being provided with a range of background knowledge to support the design process, and that is has impacted on subsequent design consulting and projects that they have since undertaken.

\section{Acknowledgements}

[optional]

\section{Disclosure statement}

No potential conflict of interest was reported by the author.

\section{Funding}

The project was funded by The Whiddon Group 


\section{Notes on contributors}

Dr Gail Kenning is a Researcher at University of Technology Sydney (UTS), Honorary Reader

in Design for Ageing and Dementia at Cardiff Metropolitan University, and Design United

Visiting Fellow at Eindhoven University of Technology. She explores how creative activity contributes to positive wellbeing through social engagement, co-design, and design research.

\section{Reference list}

'Australia to 2050'. 2010. Australia to 2050: future challenges, the 2010 intergenerational report overview. Canberra: Australian Government.

AlHW. 2013. "Ageing." Accessed 6 June 2013. http://www.aihw.gov.au/ageing/.

Alzheimer's Society. 2016a. "The dementia guide: Living well." Alzheimer's Society. https://http://www.alzheimers.org.uk/info/20105/engagement and participation/484/the live_well_with_dementia_programme.

Alzheimer's Society. 2016b. "Types of dementia." https://http://www.alzheimers.org.uk/typesofdementia.

Bang, M., and S Vossoughi. 2016. "Participatory Design Research and Educational Justice: Studying Learning and Relations Within Social Change Making." Cognition and Instruction, 34 (3):173-193. doi: https://doi.org/10.1080/07370008.2016.1181879.

Brandt, Eva. 2004. "Action research in user-centred product development." Al and Soc 18:113133. doi: DOI 10.1007/s00146-003-0271-0.

Chenoweth, Lynn, lan Forbes, Richard Fleming, Madeleine King, Jane Stein-Parbury, Georgina Luscombe, Patricia Kenny, Yun-Hee Jeaon, Marion Haas, and Henry Brodaty. 2014. "PerCEN: a cluster randomized controlled trial of person-centred residential care and environment for people with dementia." International Psychogeriatrics.

Cutler, David. 2009. Ageing artfully: Older people and professional participatory arts in the UK. United Kingdom: The Baring Foundation.

Dervin, Brenda, and Lois Foreman-Wernet. 2013. "Sense-making methodology as an approach to understanding and designing for campaign audiences: A turn to communicating communicatively." In Public communication campaigns, edited by Ronald E. Rice and Charles K Atkin. London: Sage.

Dewing, J. 2007. "Participatory reseach: A method for process consent for people who have dementia." Dementia: International journal of social research and practice 6:11-25.

Harrison, Barbara E., Gwi-Ryung Son, Jiyoung Kim, and Ann L. Whall. 2007. "Preserved Implicit Memory in Dementia: A Potential Model for Care." American journal of Alzheimer's disease and other dementias 22 (4):286-293.

Hendriks, Niels, Liesbeth Huybrechts, Andrea Wilkinson, and Karin Slegers. 2014. "Challenges in doing participatory design with people with dementia." Proceedings of the 13th 
Participatory Design Conference on Short Papers, Industry Cases, Workshop Descriptions, Doctoral Consortium papers, and Keynote abstracts - PDC '14 2:33-36.

Jonas, Wolfgang. 2014. "A cybernetic model of design research." In The Routledge Companion to Design Research. Routledge.

Kenning , Gail. 2016. Arts engagement for people with dementia: Independent evaluation of the art access program. Sydney: University of Technology Sydney and Art Gallery of News South Wales

Kenning, Gail. 2017. Making It Together: Reciprocal design to promote positive wellbeing for people living with dementia. Sydney: University of Technology Sydney.

Kenning, Gail, and Cathy Treadaway. 2017. "Conversations at the Edge of Play." Continuum 31 (6). doi: 10.1080/10304312.2017.1370075.

Kenning, Gail, and Cathy Treadaway. 2018. "Designing for Dementia: Iterative Grief and Transitional Objects." Design Issues (special issue) 34 (1):42-53. doi: https://doi.org/10.1162/DESI_a_00475.

Killick, John, and Gail Kenning. 2015. "Hand i Pockets: Creativity, playfulness and fun." Journal of dementia care 23 (5): Front page - 3.

Kinney, Jennifer M., and Clarissa A. Rentz. 2005. "Observed well-being among individuals with dementia: Memories in the making, and art program, versus other structured activity." American Journal of Alzheimer's Disease and other Dementias 20 (4):220-227.

Kitwood, Tom M. 1997. Dementia reconsidered : the person comes first, Rethinking ageing series. Buckingham England; Philadelphia: Open University Press.

Kontos, Pia C., and W Martin. 2013. "Embodiment and dementia: Exploring critical narratives of selfhood, surveillance, and dementia care." Dementia 12 (288):288-302.

Lawton, M. Powell, Kimberley Van Haitsma, and Jennifer Klapper. 1996. "Observed affect in nursing home residents with Alzheimer's disease." Journal of Gerontology 51B (1):3-14.

Maldonado Branco, Rita , Joana Quental , and Óscar Ribeiro. 2017. "Personalised participation: an approach to involve

people with dementia and their families in a

participatory design project." CoDesign 13 (2):127-143. doi: 10.1080/15710882.2017.1310903.

Manthorpe, Jill , and Kritika Samsi. 2016. "Person-centered dementia care: current perspectives." Clinical Interventions in Aging.

McClimans, Leah. 2017. Measurement in medicine : philosophical essays on assessment and evaluation. London; New York: Rowman and Littlefield International.

Mitchell, Gail J., Sherry L. Dupuis, and Pia C. Kontos. 2013. "Dementia Discourse: From imposed suffering to knowing otherwise." Journal of applied hemeneutics 12:1-19.

Mittelmark, Maurice B, and Georg F. Bauer. 2017. "The Meanings of Salutogenesis." In The Handbook of Salutogenesis edited by Maurice B. Mittelmark, Shifra Sagy, Monica Erikssonm, Georg F. Bauer, Jürgen M. Pelikan, Bengt Lindström and Geir Arild Espnes, 713. Open Access online: Springer.

National Health Scotland. 2016. Living well with dementia. Edinburgh: National Health Scotland. Norman, Donald A. 2016. "When You Come to a Fork in the Road, Take It: The Future of Design." The Journal of Design, Economics, and Innovation 2:343-348. .

Prince, Martin, Anders Wimo, Maelenn Guerchet, Gemma-Claire Ali, Yu-Tzu Wu, and Matthew Prina. 2015. World Alzheimer Report: The global impact of dementia. 
Renehan, Emma, Briony Dow, Xiaoping Lin, Irene Blackberry, Irja Haapala, Ellen Gaffy, Elizabeth Cyarto, Kathleen Brasher, and Sue Hendy. 2012. Healthy ageing literature review. edited by National ageing research institute (NARI) and Council on the ageing (COTA).

Rodgers, Paul A., and Joyce Yee. 2015. The Routledge companion to design research. New York: Routledge, Taylor \& Francis Group.

Sabat, Steven R. 2005. "Capacity for decision-making in Alzheimer's

disease: selfhood, positioning and semiotic

people." Australian and New Zealand Journal of Psychiatry 39:1030-1035.

Sabat, Steven R. 2006. "Implicit memory and people with Alzheimer's disease: Implications for caregiving." American Journal of Alzheimer's Disease and Other Dementias 21 (1):11-14. doi: http://dx.doi.org/10.1177/153331750602100113.

Stephens, Alex, Richard Cheston, and Kate Gleeson. 2012. "An exploration into the relationships people with dementia have with physical objects: An ethnographic study." Dementia 12 (6):697-712. doi: 10.1177/1471301212442585.

Treadaway, Cathy, and Gail Kenning. 2015. "Sensor e-Textiles: person centred co-design for people with later stage dementia." Working with older people 20 (2):76-85.

United_Nations,_Department_of_economic_and_Social_Affairs,_Popultion Division. 2013. World population prospects: The 2012 revision, Highlights and advance tables. .

World Health Organization. 2017. Global strategy and action plan on ageing and health. edited by World Health organization. Licence: CC BY-NC-SA 3.0 IGO.

\footnotetext{
i The term and format of the 'Meet up' is borrowed from DIY culture, 'Hack' spaces, and Maker Faires (Gauntlett, 2011; Jenkins, 2006)
} 